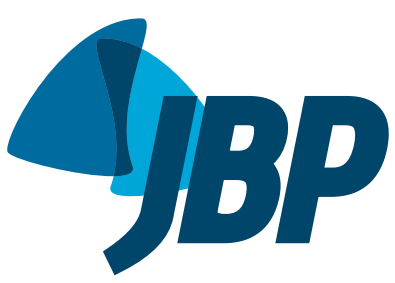

\section{Pre-operative evaluation in obstructive sleep apnea patients undergoing bariatric surgery: sleep laboratory limitations}

\author{
João Pedro Abreu Cravo', Antonio Matias Esquinas ${ }^{2}$
}

Obesity ${ }^{(1,2)}$ is a proven risk factor for obstructive sleep apnea (OSA), and it is predictable that various patients who will undergo bariatric surgery (BS) might have the disease.

However, most frequently, patients are not diagnosed with the disease whether they are symptomatic or not. Surgeons ${ }^{(2)}$ usually examine those patients in order to evaluate the risk of OSA before performing BS and might refer such patients for proper study and treatment.

Duarte and Magalhães-da-Silveira(1) ${ }^{(1)}$ conducted a retrospective study aiming to evaluate and identify which parameters could predict a higher chance of OSA in that specific population. They also developed a 6-item score that had good accuracy for the diagnosis of moderate and severe OSA.

We understand the limitations of the study; however, we would like to consider some key aspects that need to be taken into account for a proper clinical extrapolation.

First, hypertension ${ }^{(3)}$ is known to be an important factor in the development of OSA. ${ }^{(4)}$ There are widely available data revealing the benefits of BS in blood pressure control. In that study, ${ }^{(1)}$ we found that hypertension was not considered a predictive factor in their group of patients. This might have been due to the fact that patients being treated for hypertension or not were grouped together, which might have influenced the results.

Second, the authors referred that they were not able to address all known comorbidities or other sleep complaints. Considering that the article is about OSA patients awaiting BS, we think that there is a potentially relevant predictive factor that was not fully explored: metabolic syndrome, ${ }^{(4)}$ which is consistently associated with both OSA and BS. We believe that, even if we exclude hypertension, cholesterol levels, and other comorbidities, such as diabetes, the metabolic syndrome might predict OSA and must be taken into account in future studies.

Finally, we know that it is necessary to perform OSA screening in all patients who will undergo this type of surgery, regardless of any score. We do not want to undermine the value or the clinical importance of the NO-OSAS score; however, we question whether there is any advantage to its routine application. Would it change our behavior?

In our opinion, that article ${ }^{(1)}$ demonstrates that it is possible to perceive which subgroups of this specific population are at a higher risk in order to direct our attention to them. However, other important clinical parameters need to be assessed so that its predictive power can be enhanced.

\title{
REFERENCES
}

1. Duarte RL, Magalhães-da-Silveira FJ. Factors predictive of obstructive sleep apnea in patients undergoing pre-operative evaluation for bariatric surgery and referred to a sleep laboratory for polysomnography. J Bras Pneumol. 2015;41(5):440-8. http://dx.doi.org/10.1590/S180637132015000000027

2. Selim BJ, Surani SR, Ramar K. Role of preoperative screening for adult patients for obstructive sleep apnea. Hosp Pract (1995). 2014;42(5):100-

7. http://dx.doi.org/10.3810/hp.2014.12.1163

3. Torres G, Sánchez-de-la-Torre M, Barbé F. Relationship Between OSA and Hypertension. Chest. 2015;148(3):824-32. http://dx.doi.org/10.1378/ chest.15-0136

4. Bonsignore MR, Borel AL, Machan E, Grunstein R. Sleep apnoea and metabolic dysfunction. Eur Respir Rev. 2013;22(129):353-64. http:// dx.doi.org/10.1183/09059180.00003413 


\title{
Authors' reply
}

\author{
Ricardo Luiz de Menezes Duarte ${ }^{1,2}$, Flavio José Magalhães-da-Silveira ${ }^{1}$
}

In response to questions raised about our study, ${ }^{(1)}$ we would like to make some comments. The literature indicates that there is a bidirectional association between obstructive sleep apnea (OSA) and systemic arterial hypertension (SAH). ${ }^{(2)}$ With regard to the role of $\mathrm{SAH}$ and OSA as risk factors for the development of OSA and $\mathrm{SAH}$, respectively, some data merit special attention: OSA as a causal risk factor for the development of SAH has been more extensively studied than has the reverse; in addition, the treatment of OSA with continuous positive airway pressure usually improves blood pressure levels in patients with hypertension. (2) In contrast, the treatment of SAH with antihypertensives in order to improve OSA yields conflicting results; except, perhaps, for the treatment of SAH with diuretics, especially spironolactone, because it reduces parapharyngeal edema and secondary airway obstruction. (2)

With regard to whether or not patients were being treated, we do not believe that this would have influenced the magnitude of association between $\mathrm{SAH}$ and OSA, because the main clinical questionnaires that use the variable SAH do not discriminate between patients who are on antihypertensive treatment and those who are not. (3) In our study, (1) SAH was found to be a prognostic factor in the univariate analysis at three different cut-off points-apnea-hypopnea index $(\mathrm{AHI}) \geq 5$ events/h; $\mathrm{AHI} \geq 15$ events/h; and $\mathrm{AHI} \geq$ 30 events/h-however, when used in the multivariate analysis, SAH was found to be an independent prognostic factor only at the cut-off point of $\mathrm{AHI} \geq 5$ events/h, showing that, in the more severe forms of OSA, SAH acts as a confounding factor, which is why it was not used in our questionnaire.

Metabolic syndrome is defined as the presence of three or more of the following five factors: 1) waist circumference $>80 \mathrm{~cm}$ in women and $>94 \mathrm{~cm}$ in men; 2 ) serum triglycerides $\geq 150 \mathrm{mg} / \mathrm{dL}$ (or on drug treatment for elevated triglycerides); 3) HDL cholesterol $<40 \mathrm{mg} / \mathrm{dL}$ in men and $<50 \mathrm{mg} / \mathrm{dL}$ in women (or on drug treatment for reduced HDL cholesterol); 4) SAH (or on antihypertensive drug treatment); and 5) fasting glucose $\geq 100 \mathrm{mg} / \mathrm{dL}$ (or on drug treatment for elevated glucose). ${ }^{(4)}$ Metabolic syndrome was not used in our questionnaire because its confirmation requires serum level determinations, which would make it impossible to produce an easy-to-administer questionnaire for physicians and patients.

The aim in designing a clinical questionnaire is not to replace polysomnography (PSG). The aim of a questionnaire is to screen patients, selecting those at high risk and enabling faster and cheaper diagnosis with portable monitoring devices. ${ }^{(3)}$ Type I PSG, despite being the gold standard for the diagnosis of OSA, has its inherent costs and is associated with long waiting lists. Since the use of portable methods has been validated in the bariatric population, ${ }^{(5)}$ our questionnaire, specifically developed for this population, can indeed change practice, selecting high-risk patients for home diagnosis, limiting costs, decreasing the waiting time for surgery, and decreasing the waiting time for laboratory PSG.

\section{REFERENCES}

1. Duarte RL, Magalhães-da-Silveira FJ. Factors predictive of obstructive sleep apnea in patients undergoing pre-operative evaluation for bariatric surgery and referred to a sleep laboratory for polysomnography. J Bras Pneumol. 2015;41(5):440-8. http://dx.doi. org/10.1590/S1806-37132015000000027

2. Torres G, Sánchez-de-la-Torre M, Barbé F. Relationship between OSA and hypertension. Chest. 2015;148(3):824-32. http://dx.doi. org/10.1378/chest.15-0136

3. Abrishami A, Khajehdehi A, Chung F. A systematic review of screening questionnaires for obstructive sleep apnea. Can J Anesth. 2010;57(5):423-38. http://dx.doi.org/10.1007/s12630-010-9280-x

4. Alberti KG, Eckel RH, Grundy SM, Zimmet PZ, Cleeman JI, Donato $K A$, et al. Harmonizing the metabolic syndrome: a joint interim statement of the International Diabetes Federation Task Force on Epidemiology and Prevention; National Heart, Lung, and Blood Institute; American Heart Association; World Heart Federation; International Atherosclerosis Society; and International Association for the Study of Obesity. Circulation. 2009;120(16):1640-5. http:// dx.doi.org/10.1161/CIRCULATIONAHA.109.192644

5. Oliveira MG, Treptow EC, Fukuda C, Nery LE, Valadares RM, Tufik $S$, et al. Diagnostic accuracy of home-based monitoring system in morbidly obese patients with high risk for sleep apnea. Obes Surg 2015;25(5):845-51. http://dx.doi.org/10.1007/s11695-014-1469-6 